University of South Carolina

Scholar Commons

$2-1-2010$

\title{
Adhesion at Diamond /Metal Interfaces: A Density Functional Theory Study
}

Haibo Guo

Yue Qi

Xiaodong Li

University of South Carolina - Columbia, lixiao@cec.sc.edu

Follow this and additional works at: https://scholarcommons.sc.edu/emec_facpub

Part of the Heat Transfer, Combustion Commons, and the Other Mechanical Engineering Commons

\section{Publication Info}

Published in Journal of Applied Physics, Volume 107, Issue 3, 2010, pages \#033722-.

(C) Journal of Applied Physics 2010, American Institute of Physics.

Guo, H. \& Li, X. (1 February 2010). Adhesion at Diamond /Metal Interfaces: A Density Functional Theory Study. Journal of Applied Physics, 107 (3), \#033722. http://dx.doi.org/10.1063/1.3277013

This Article is brought to you by the Mechanical Engineering, Department of at Scholar Commons. It has been accepted for inclusion in Faculty Publications by an authorized administrator of Scholar Commons. For more information, please contact digres@mailbox.sc.edu. 


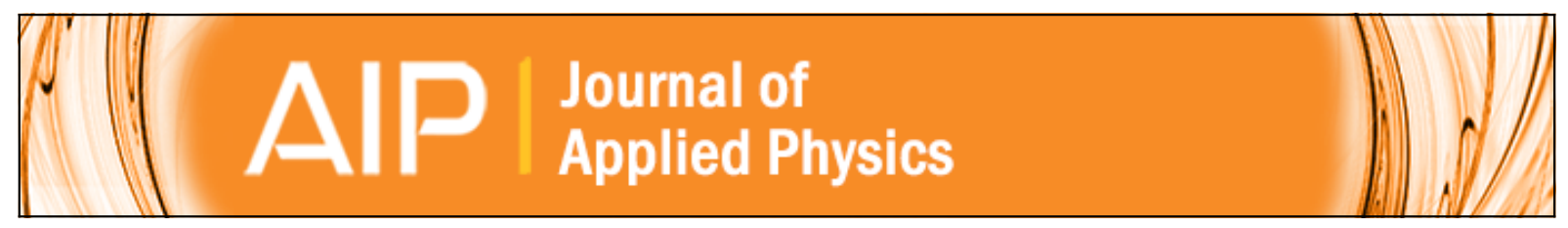

\section{Adhesion at diamond/metal interfaces: A density functional theory study}

Haibo Guo, Yue Qi, and Xiaodong Li

Citation: Journal of Applied Physics 107, 033722 (2010); doi: 10.1063/1.3277013

View online: http://dx.doi.org/10.1063/1.3277013

View Table of Contents: http://scitation.aip.org/content/aip/journal/jap/107/3?ver=pdfcov

Published by the AIP Publishing

\section{Articles you may be interested in}

Qualitative link between work of adhesion and thermal conductance of metal/diamond interfaces

J. Appl. Phys. 115, 123509 (2014); 10.1063/1.4869668

Cu adhesion on tantalum and ruthenium surface: Density functional theory study

J. Appl. Phys. 107, 103534 (2010); 10.1063/1.3369443

Coating geometries of metals on single-walled carbon nanotubes

Appl. Phys. Lett. 96, 063108 (2010); 10.1063/1.3309752

Study of hardness and deformation of brittle materials with a density functional theory

J. Appl. Phys. 104, 053508 (2008); 10.1063/1.2968226

Experimental studies on relationships between the electron work function, adhesion, and friction for $3 \mathrm{~d}$ transition metals

J. Appl. Phys. 95, 7961 (2004); 10.1063/1.1738531
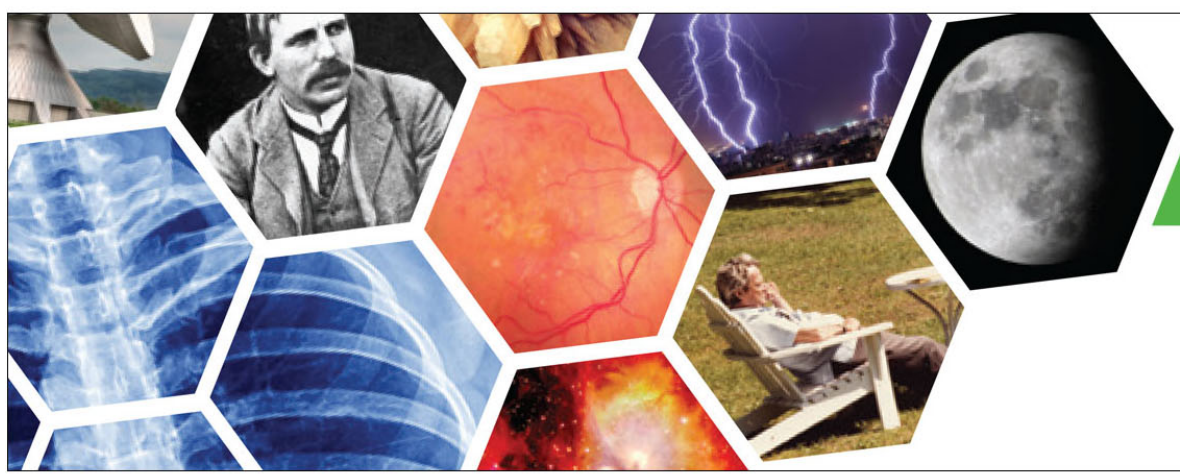

\section{SUBSCRIBE TO \\ physics today}




\title{
Adhesion at diamond/metal interfaces: A density functional theory study
}

\author{
Haibo Guo, ${ }^{1}$ Yue Qi, ${ }^{2, a)}$ and Xiaodong $\mathrm{Li}^{1}$ \\ ${ }^{1}$ Department of Mechanical Engineering, University of South Carolina, 300 Main Street, Columbia, South \\ Carolina 29208, USA \\ ${ }^{2}$ Materials and Processes Laboratory, General Motors R\&D Center, 30500 Mound Road, Warren, Michigan \\ 48090, USA
}

(Received 17 March 2009; accepted 26 November 2009; published online 12 February 2010)

\begin{abstract}
To understand the basic material properties required in selecting a metallic interlayer for enhanced adhesion of diamond coatings on the substrates, the interfaces between diamond and metals with different carbide formation enthalpies $(\mathrm{Cu}, \mathrm{Ti}$, and $\mathrm{Al})$ are studied using density functional theory. It is found that the work of separation decreases, while the interface energy increases, with the carbide formation enthalpy $\Delta H_{f}(\mathrm{Ti}<\mathrm{Al}<\mathrm{Cu})$. By comparing the work of separation at the interface with the work of decohesion of the metal, we found that the fracture is more likely to initiate in the metal phase near the interface; therefore a metal phase with a larger surface energy, $\gamma_{s}(\mathrm{Ti}>\mathrm{Cu}>\mathrm{Al})$, is needed to achieve a higher overall interface strength. In addition, when the surface energy is larger than the interface energy, a wetted diamond/metal interface is formed during diamond nucleation, providing the strongest adhesion compared to other growth modes. These results indicate that a strong carbide-forming ability and a large surface energy of the interlayer promote nucleation and enhance the adhesion and interface strength of the coating/substrate system. () 2010 American Institute of Physics. [doi:10.1063/1.3277013]
\end{abstract}

\section{INTRODUCTION}

Chemical vapor deposited (CVD) diamond coatings have various applications in many fields, including tribology, mechanics, electronics, ${ }^{1}$ optics, ${ }^{2}$ and micro- $/$ nanoelectromechanical systems (MEMS/NEMS), ${ }^{3,4}$ because of their unique properties. However, the broader applications of diamond coatings are hindered by their poor adhesion to substrates. For example, the adhesion failure is a key challenge for integrating diamond films with other materials in diamond-based MEMS/NEMS devices, ${ }^{5}$ and for using it as the protective coatings in cutting tools that are under high and complicated external stresses during machining. In some electronic devices, metals are deposited on diamonds, where the adhesion of diamond/metal interfaces also influences the electronic contact between metals and the semiconductive diamond. $^{6}$

The poor adhesion of diamond coatings has been attributed to the high residual stress in the coatings and the weak interfaces between the coatings and substrates. In addition to reducing the residual stresses in diamond coatings, ${ }^{7,8}$ many efforts, including preseeding, scratching, and adding an interlayer, have been made to enhance the interfacial adhesion of the diamond/metal systems. ${ }^{9}$ Adding an appropriate interlayer between the diamond coating and substrate can effectively improve the interfacial adhesion. ${ }^{9}$ The interlayer is expected to serve the following three major functions: (1) as an adhesive layer to enhance the bonding strength of the coating to the substrate, (2) as a diffusion barrier to shield off the detrimental elements such as Co (typically used as a binder for cemented tungsten carbide), which catalyzes diamond to

\footnotetext{
a) Author to whom correspondence should be addressed. Electronic mail: yue.qi@gm.com.
}

graphite and consequently leads to the coating delamination, and (3) as a nucleation layer to facilitate diamond nucleation and growth in the CVD process. In searching for the best interlayer materials, previous studies suggested that carbideforming metals (e.g., $\mathrm{Ti}$ and $\mathrm{Cr}$ ) favor diamond nucleation and adhesion more than non-carbide-forming metals (e.g., $\mathrm{Cu}$ and $\mathrm{Au}){ }^{9}$ It is generally believed that the formation of carbides indicates good chemical affinity between the material and carbon, and furthermore, the transition carbide layer provides strong chemical bonds for the diamond coating and the metallic interlayer. However, this theory is difficult to prove due to the lack of quantitative measurements of the true adhesion or interface toughness of diamond/metals. Although different semiquantitative methodologies, which are essentially destructive, have been developed to characterize the adhesion, including Rockwell $\mathrm{C}$ indentation, ${ }^{10}$ scratch, ${ }^{11}$ superlayer, ${ }^{12}$ bulge, ${ }^{13}$ and blister tests, ${ }^{14}$ the true adhesion energy cannot be precisely measured by experiments. In contrast, accurate atomistic simulation can provide unambiguous inputs for macroscale mechanical modeling. ${ }^{15,16}$ In this study, we use atomistic computational modeling and simulations to investigate the adhesion of diamond/metal interfaces.

The adhesion of a coating/substrate system is generally expressed in terms of energies to detach the coating from its substrate by an external load. ${ }^{17,18}$ The energies of detachment include the intrinsic work of adhesion, and extrinsic energies that are associated with dissipative and irreversible processes such as plastic deformation and interface roughness. ${ }^{17,19}$ The intrinsic work of adhesion is of key importance since many of the irreversible energies can also scale with it. ${ }^{17}$ This study aims to seek for the connection between the intrinsic interfacial adhesion, which is represented by the ideal work of separation $\left(W_{\text {sep }}\right)$, and the formation enthalpy of the carbide phase $\left(\Delta H_{f}\right)$ of a metallic interlayer to provide guide- 
lines for the selection for interlayer materials. Here, we take $\mathrm{Al}, \mathrm{Cu}$, and $\mathrm{Ti}$ as model materials. Of the three model materials, $\mathrm{Cu}$ does not form any carbide, while $\mathrm{Al}$ and $\mathrm{Ti}$ form $\mathrm{Al}_{4} \mathrm{C}_{3}$ and TiC, respectively. Since $\mathrm{Cu}$ has an almost perfect lattice match with diamond, there were many attempts to grow CVD diamond epitaxially on $\mathrm{Cu}$. However, this goal has never been achieved without preseeding diamond on the surface. ${ }^{20,21}$ It was found that an intermediate graphite layer forms on the $\mathrm{Cu}$ substrate before diamond nucleates. ${ }^{22-24}$ Surface pretreatments such as carbon implantation and laserirradiation result in the formation of a graphite interlayer, preventing the epitaxial growth of diamond on $\mathrm{Cu}^{25,26}$ The diamond/Ti interface was found to be tough and adherent. ${ }^{27}$ No report is found on CVD diamond growth on $\mathrm{Al}$, although Al can form carbides.

The formation of carbides is a spontaneous process in the deposition of diamond by CVD. A sharp diamond/metal interface is seldom observed for carbide-forming metals, in which case the interface region becomes diamond/carbide/ metal. It seems unrealistic to model a sharp interface between diamond and metals. However, such a sharp interface keeps the comparison on both carbide-forming and noncarbide-forming metals consistent. In addition, the formation of carbide phases can be suppressed and sharp diamond/ metal interfaces can be achieved by depositing metal coatings on diamond substrates at low temperatures using physical vapor deposition for carbide-forming metals. The interfaces between diamond and $\mathrm{Cu}, \mathrm{Al}$, and $\mathrm{Ti}$ are of interest for diamond-based electronic devices. ${ }^{28}$ Epitaxial growth of $\mathrm{Cu}$ was reported on clean as well as contaminated diamond surfaces. ${ }^{6,29} \mathrm{Al}$ is also used as a gate interfacial layer in diamond field-effect transistors. ${ }^{30}$ Theoretical studies using atomically sharp interfaces have confirmed that the interface between contaminated, especially hydrogen-terminated, diamond and $\mathrm{Al}$ or $\mathrm{Cu}$ is much weaker than clean diamond/ metal interfaces. ${ }^{16,31}$ Recent experiments on Ti interlayer show that cracks can occur inside the TiC phase, but stop at the $\mathrm{Ti} / \mathrm{TiC}$ and diamond/TiC interfaces, which have lower theoretical adhesion properties compared to the sharp diamond/Ti interface. Thus the computed adhesion properties using sharp diamond/metal interface models would serve as the upper limits of diamond coatings on metallic interlayers, and provide guidance for interlayer selection to improve the adhesion of diamond coatings.

In addition to the $W_{\text {sep }}$ at the interface, it is important to know how the interfacial strength compares with the cohesion strength of the constituent materials. The influence of the diamond/metal interface bonds can spread away from the interface by short range redistribution of charge densities. This may affect the failure mode of the overall interface system because the fracture is not always initiated right at the interface, but at wherever the weakest location. The comparison of the $W_{\text {sep }}$ at the interface and the work of decohesion, $W_{\text {dec }}$, in the weaker material (metal in this case) near the interface will enable us to predict precisely the fracture location under an applied tensile load. Electronic structure will be also analyzed to reveal the charge density redistribution due to the formation of the interfacial bonds, and this will provide additional insights into the adhesion properties of the diamond/metal interfaces.

Another role of the interlayer in diamond coating/ substrate is to promote the nucleation and growth of diamond. The nucleation of diamond on a substrate in the CVD process involves the formation of a new diamond/metal interface at the substrate surface. The interface energy here describes the energy barrier of the formation of the interface, which is related to the nucleation process where individual atoms or molecules agglomerate at a foreign surface and form a new interface. The interface energy and its correlation with $\Delta H_{f}$ will be calculated, and then its role in the nucleation of diamond coatings and the implication to the macroscopic adhesion properties will be discussed.

\section{COMPUTATIONS}

The ab initio calculations are based on the density functional theory (DFT) implemented in the Vienna ab initio simulation package (VASP). ${ }^{32,33}$ In the calculations, the ionelectron interaction is described by projector augmented wave potentials, ${ }^{34}$ and the exchange-correlation functional is generalized gradient approximation by Perdew and Wang. ${ }^{35}$ The plane-wave energy cutoff is $400.0 \mathrm{eV}$ for all the three diamond/metal interfaces based on convergence tests. For bulk metal calculations, the integration is computed over an $11 \times 11 \times 11 k$-point mesh that results in 56 irreducible $k$-points in the Brillouin zone for fcc $\mathrm{Al}$ and $\mathrm{Cu}$, and 96 for hcp Ti. For surface and interface structures, a gammacentered $8 \times 8 \times 1$ mesh is employed and the resultant irreducible $k$-points in the Brillouin zone are 12 for diamond/Al, 10 for diamond $/ \mathrm{Cu}$, and 21 for diamond/Ti interfaces. The positions of atoms are relaxed to minimize the free energy using the conjugate gradient method until the HellmannFeynman force on each atom is less than $0.05 \mathrm{eV} / \AA$. The convergence tolerance for the electronic relaxation is $10^{-5} \mathrm{eV} /$ cell. The linear tetrahedron method with Blöchl corrections is used to calculate the total energy. More computational details for $\mathrm{Al}$ and diamond/Al interfaces have been stated in Ref. 31. As a validation of the computational methodology, all the computed bulk lattice constants are in good agreement with experimental values, within $1.3 \%$.

For the three metals selected, the closed packed surfaces are modeled by slabs in vacuum supercells. Every slab has periodic boundary conditions along all the three directions, and the two free surfaces are separated by a $10 \AA$ thick vacuum slab to preclude interactions from image surfaces. All the atoms are allowed to relax. The surface energy is derived by least squares fitting to the total energy as a function of the numbers of layers in the slab. The thickness of each slab is determined through a convergence test. As depicted in Table I, five layers of $\mathrm{Al}(111)$, five layers of $\mathrm{Cu}(111)$, and four layers of $\mathrm{Ti}(0002)$ are sufficient for the surface energy to converge within $0.01 \mathrm{~J} / \mathrm{m}^{2}$. The calculated $\mathrm{Al}(111)$ surface energy, $0.78 \mathrm{~J} / \mathrm{m}^{2}$, compares well with other DFT results of $0.91 \mathrm{~J} / \mathrm{m}^{2}$ (Ref. 36) and $0.83 \mathrm{~J} / \mathrm{m}^{2}$ (Ref. 37) using local density approximation (LDA), and $0.75 \mathrm{~J} / \mathrm{m}^{2}$ using generalized gradient approximation (GGA). ${ }^{36}$ The re- 
TABLE I. The surface energy convergence with the slab thickness for Al (111), $\mathrm{Cu}$ (111), and Ti (0001) surfaces.

\begin{tabular}{lcc}
\hline \hline System & No. of layers & $\begin{array}{c}\text { Surface energy } \\
\left(\mathrm{J} / \mathrm{m}^{2}\right)\end{array}$ \\
\hline $\mathrm{Al}(111)$ & 3 & 0.75 \\
& 5 & 0.78 \\
$\mathrm{Cu}(111)$ & $3-4$ & 1.28 \\
& $5-7$ & 1.29 \\
& $7-10$ & 1.29 \\
$\mathrm{Ti}(0001)$ & $3-7$ & 1.92 \\
& $8-9$ & 1.92 \\
\hline
\end{tabular}

laxed $\mathrm{Cu}(111)$ surface energy, $1.29 \mathrm{~J} / \mathrm{m}^{2}$, agrees with $1.32 \mathrm{~J} / \mathrm{m}^{2}$ (Ref. 38) and $1.28 \mathrm{~J} / \mathrm{m}^{2}$ (Ref. 39) calculated using GGA, $1.585 \mathrm{~J} / \mathrm{m}^{2}$ using a mixed-basis pseudopotential method, ${ }^{40}$ and $1.41 \mathrm{~J} / \mathrm{m}^{2}$ by a full-potential linearized augmented plane-wave (FP-LAPW) method. ${ }^{36}$ The surface energy of $\mathrm{Ti}(0001), 1.92 \mathrm{~J} / \mathrm{m}^{2}$, agrees well with $1.99 \mathrm{~J} / \mathrm{m}^{2}$ calculated using FP-LAPW (Ref. 36) and $1.96 \mathrm{~J} / \mathrm{m}^{2}$ by Green function method. ${ }^{41}$ In short, the calculated surface energies are in good agreement with the reported values calculated by other methodologies, and will be used in the subsequent interface models.

Based on the convergence tests in the surface energy calculations, ten layers of $\mathrm{Cu}(111)$ and $\mathrm{Al}(111)$, and nine layers of $\mathrm{Ti}(0002)$ are selected to form the diamond/metal interface with six bilayers of diamond(111)-1 $\times 1$. In a periodic cell setting without vacuum, each unit cell contains two identical interfaces. For all the interface structures, we choose the interface orientations with the least mismatch strain (less than $1.5 \%$, which was estimated to cause less than $\sim 5 \%$ error in the work of separations ${ }^{42}$ ). At this level of mismatch, the separation between misfit dislocations in a realistic interface structure is far enough so that the periodic interface is a reasonable approximation. ${ }^{43}$ The lattice mismatch for diamond and $\mathrm{Cu}$ is $1.3 \%$; thus the diamond(111)/ $\mathrm{Cu}(111)$ interface has one atom per layer in a rhombohedral cell. The common cell length is $2.538 \AA$ after minimizing the stress of the simulation cell. With the compromised lattice, diamond is under $0.6 \%$ tensile strain while copper is under $0.7 \%$ compressive strain. We have tested interface registries for which $\mathrm{Cu}$ atoms are at on-top, bridge, and hollow sites relative to carbon atoms, and found that the on-top registry has the lowest energy. The registry is referred to as diamond $(111)\langle 2 \overline{1} \overline{1} 0\rangle \| \mathrm{Cu}(111)\langle 2 \overline{1} \overline{1} 0\rangle$. There are $10 \mathrm{Cu}$ and 12 carbon atoms in each unit cell. Due to the large lattice mismatch (14.0\% for diamond/Al and $17.0 \%$ for diamond/ Ti) between the unit cell length of diamond(111) and $\mathrm{Ti}(0002)$ or $\mathrm{Al}(111)$, a supercell is created to match four carbon atoms in diamond(111) with three $\mathrm{Ti}$ atoms in $\mathrm{Ti}(0002)$ or three $\mathrm{Al}$ atoms in $\mathrm{Al}(111)$ per layer, with a rotation of the metal phase by $30^{\circ}$ around the common [0001] to reduce the interface mismatch to $-1.3 \%$ for diamond(111)/ $\mathrm{Al}(111)$ and $1.3 \%$ for diamond(111)/Ti(0002). The resultant metals ( $\mathrm{Ti}$ and $\mathrm{Al})\langle 01 \overline{1} 0\rangle$ overlap with diamond $\langle 2 \overline{1} \overline{10}\rangle$, and the two interfaces are referred to as diamond $(111)\langle 2 \overline{1} \overline{1} 0\rangle \| \operatorname{Ti}(0002)\langle 01 \overline{1} 0\rangle$ and diamond $(111)\langle 2 \overline{1} \overline{1} 0\rangle \| \operatorname{Al}(0002)\langle 01 \overline{1} 0\rangle$, respectively. This registry was found to have the lowest energy among other tested registries. ${ }^{31}$ The unit cell contains $27 \mathrm{Ti}$ and 48 carbon atoms with the compromised cell edge length $a=b$ $=5.048 \AA$ for the diamond/Ti model, and $30 \mathrm{Al}$ and 48 carbon atoms with $a=b=5.027 \AA$ for the diamond/Al model. In all cases, the interfaces between periodic images are made identical to preclude any dipole moment in the cell.

The interface models are subjected to geometry optimizations and energy minimizations to obtain the equilibrium structures. The average distances at the interfaces are found to be $1.86 \AA$ between diamond and Al, $2.06 \AA$ between diamond and $\mathrm{Cu}$, and $1.94 \AA$ between diamond and Ti. For all the systems, the distances are shorter than the planer distances in equilibrium bulk metals: $2.33 \AA$ for $\mathrm{Al}(111), 2.10 \AA$ for $\mathrm{Cu}(111)$, and $2.32 \AA$ for $\mathrm{Ti}(0002)$, respectively. This implies that the interaction between the carbon atoms and the metal atoms may be stronger than that between the metal atoms. More detailed calculation results of the work of separations and analysis to the electronic structure will further confirm this observation, in the Secs. III and IV.

\section{INTERFACE STRENGTH}

\section{A. Ideal work of separation at the interface}

The ideal work of separation is the reversible work required to separate the interface into free surfaces, neglecting plastic and diffusional degrees of freedom. It is defined as ${ }^{18}$

$$
W_{\text {sep }}=\gamma_{1 V}+\gamma_{2 V}-\gamma_{\text {int }},
$$

where $\gamma_{1 V}$ and $\gamma_{2 V}$ are the surface energies of slabs 1 and 2, respectively; and $\gamma_{\text {int }}$ is the interface energy. In the slab model, the $W_{\text {sep }}$ is calculated as $W_{\text {sep }}=\left(E_{1}^{\mathrm{tot}}+E_{2}^{\mathrm{tot}}-E_{12}^{\mathrm{tot}}\right) / A$ where $E_{i}^{\mathrm{tot}}$ is the total energy of constituent slab $i, E_{12}^{\mathrm{tot}}$ is the total energy of the interface system, and $A$ is the interface area. The shape and volume of the supercell remain the same for separate slabs and the interface system to obtain a consistent comparison.

$W_{\text {sep }}$ is directly related to the interface toughness. In Griffith fracture theories, ${ }^{44-46}$ the critical stress $\sigma_{f}^{G}$ for a sharp planar crack of length $2 c$ is a function of Young's modulus $E$ and of the crack resistance $\gamma_{s}$ as follows:

$$
\sigma_{f}^{G}=\sqrt{\frac{2 \gamma_{s} E^{\prime}}{\pi c}}=\sqrt{\frac{W_{\mathrm{sep}} E^{\prime}}{\pi c}},
$$

where $E^{\prime}=E /\left(1-v^{2}\right)$ with the Poisson ratio of $v \cdot \gamma_{s}$ is usually identified with the free surface energy of the cleavage surface. But for an asymmetric crack at the interface, $2 \gamma_{s}$ is replaced with $W_{\text {sep. }}$. Thus, $W_{\text {sep }}$ is an indicator of the crack resistance and the toughness of the interface. From Eq. (2) we see a large $\sigma_{f}^{G}$ or $W_{\text {sep }}$ is desirable for diamond/metal interfaces, such as those in cutting tools that work under large stresses. Table II lists the $W_{\text {sep }}$ of diamond $/ \mathrm{Cu}$, diamond/Ti, and previously calculated diamond/Al interfaces. ${ }^{31}$ The calculated $W_{\text {sep }}$ for the diamond/Cu interface, $3.36 \mathrm{~J} / \mathrm{m}^{2}$, agrees with $3.76 \mathrm{~J} / \mathrm{m}^{2}$ using the FP-LAPW 
TABLE II. The ideal works of separation, $W_{\text {sep }}$, and the interface energies, $\gamma_{\text {int }}$, of the diamond/metal interfaces; the works of decohesion, $W_{\text {dec }}$, inside the metals; and the surface energies, $\gamma_{s}$, of the metals. Unit: $\mathrm{J} / \mathrm{m}^{2}$. (0|1) represents the crack occurs right at the diamond/metal interface, (1|2) represents the crack occurs in the metal between the first and second atomics layers, counted from the interface, etc.

\begin{tabular}{lccc}
\hline & Diamond/Al & Diamond/Cu & Diamond/Ti \\
\hline$W_{\text {sep }}(0 \mid 1)$ & 4.08 & 3.36 & 5.77 \\
$W_{\text {dec }}(1 \mid 2)$ & 2.25 & 2.60 & 4.01 \\
$W_{\text {dec }}(2 \mid 3)$ & 1.56 & 2.60 & 3.71 \\
$W_{\text {dec }}(3 \mid 4)$ & 1.60 & 2.89 & 3.88 \\
$2 \gamma_{s}$ & 1.56 & 2.58 & 3.84 \\
$\gamma_{\text {int }}$ & 2.45 & 3.58 & 1.88 \\
\hline \hline
\end{tabular}

method in Ref. 16. The order of $W_{\text {sep }}$, ranking from large to small, is diamond/Ti $\left(5.77 \mathrm{~J} / \mathrm{m}^{2}\right)>$ diamond/ Al $\left(4.08 \mathrm{~J} / \mathrm{m}^{2}\right)>$ diamond $/ \mathrm{Cu}\left(3.36 \mathrm{~J} / \mathrm{m}^{2}\right)$. This is consistent with the general belief that carbide-forming metals have better adhesion to diamond coatings than non-carbideforming metals, although there is no intermediate carbide layer in the sharp diamond/metal interface model.

\section{B. Bonding nature at the interface}

The electron localization function (ELF) (Ref. 47) is employed to explore the interfacial bond character in the three systems. ELF is a localized function of same-spin pairs, which is interpreted either as a spherical average of the Hartree-Fock same-spin pair probability ${ }^{47}$ or the excess local kinetic energy due to Pauli exclusion principle. ${ }^{48}$ The value of ELF ranges from 0 to 1, where 1 corresponds to the localization as in covalent bonds, and 0.5 corresponds to the electron-gas-like pair probability as in metallic bonds. ${ }^{47}$ Topology analysis to ELF can effectively reveal various chemical bonds. Figures 1 and 2 show the ELF contour plots of the three optimized diamond/metal interfaces. The cross sections along the diamond (110) planes that are perpendicular to the interfaces and passing through the supercell origins are shown in Fig. 1. In Fig. 1, atomic positions on the cross sections are marked as well. Notice that atoms at two of every three $\mathrm{Al}(111)$ planes and one of every two $\mathrm{Ti}(0002)$ atomic planes are not on this cross section. The planes that are parallel to the interfaces and between the diamond and the metal layers are shown in Fig. 2. Figures 2(a)-2(c) also show the projections of the carbon and metal atoms on the interfaces. The interface registry for diamond/Al and diamond/Ti is schematically shown as Fig. 2(d) to assist visualizing the atomic positions and directions. The three Al and $\mathrm{Ti}$ atoms in the supercell are numbered, according to their different neighboring carbon atoms: atom 1 is directly on top of the first layer of carbon atom, atom 2 is on the hollow site of the top layer carbon but on top of the second carbon layer, and atom 3 is on top of the hollow set of both the first and the second carbon layers.

Figure 1 shows that covalent bonds (indicated by the regions of red (dark gray) colors) are located between neighboring carbon atoms and between the carbon atoms and the metal atoms at the interfaces. Figure 2 shows that the strongest covalent interfacial bond is between the carbon atom and the metal atom directly atop it. For the diamond/Al and diamond/Ti interfaces, covalent bonds are also formed by sharing the two metal atoms on top of the hollow sites (atoms 2 and 3) with the three underlying carbon atoms. Thus there are two types of covalent bonds at the diamond/Al and diamond/Ti interfaces: (1) the bonds between the carbon atoms and the atop metal atoms, and (2) the bonds between the carbon atoms and the metal atoms on top of the hollow sites. Because of the one-to-one match between diamond(111) and $\mathrm{Cu}(111)$, there are only type (1) bonds at the diamond/ $\mathrm{Cu}$ interface. The contour plots in Fig. 1 indicate that the maximum ELF value at the region between carbon and atop $\mathrm{Cu}$ is around 0.7 , which is smaller than that between carbon and $\mathrm{Al}$ or $\mathrm{Ti}$, about $0.8-0.9$. This means that the covalent $\mathrm{Cu}-\mathrm{C}$ bond is weaker than the Al-C and Ti-C bonds. For the interfaces of diamond/Al and diamond/Ti, the maximum ELF value of type (1) covalent bonds is larger than that of type (2) bonds, which means that type (1) covalent bonds are stronger than type (2). The maximum ELF value of type (1) bonds in diamond/Al is very similar to that in diamond/Ti; both are about 0.9 . By carefully examining the contour plots in Fig. 2, we find that the maximum ELF value of type (2) in diamond/Al is about 0.8 , slightly lower than that in diamond/ Ti, about 0.9. This indicates that the covalent bonds in diamond/Al are weaker than that in diamond/Ti.
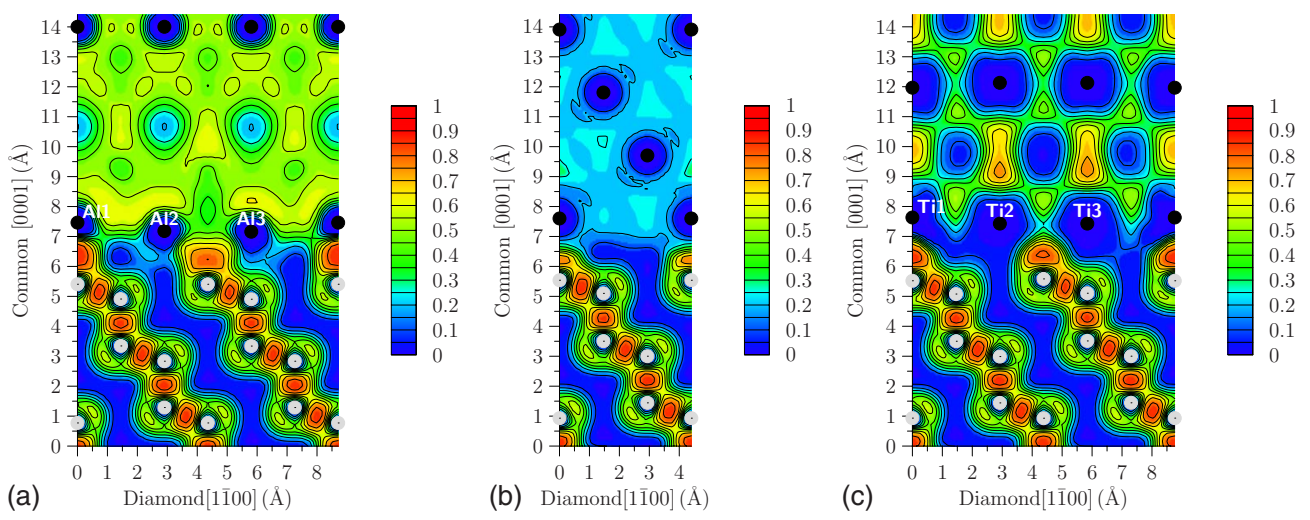

FIG. 1. (Color online) ELF contour plots along diamond (110) plane. The filled gray circles are carbon atoms, and the filled black circles in (a), (b), and (c) are $\mathrm{Al}, \mathrm{Cu}$, and $\mathrm{Ti}$ atoms, respectively. Note that two $\mathrm{Al}(111)$ atomic planes in (a) and one Ti (0002) atomic plane in (c) are not shown because of the rotations of metals to match the diamond (111) plane. Each neighboring color represents a difference of 0.1 in ELF. 

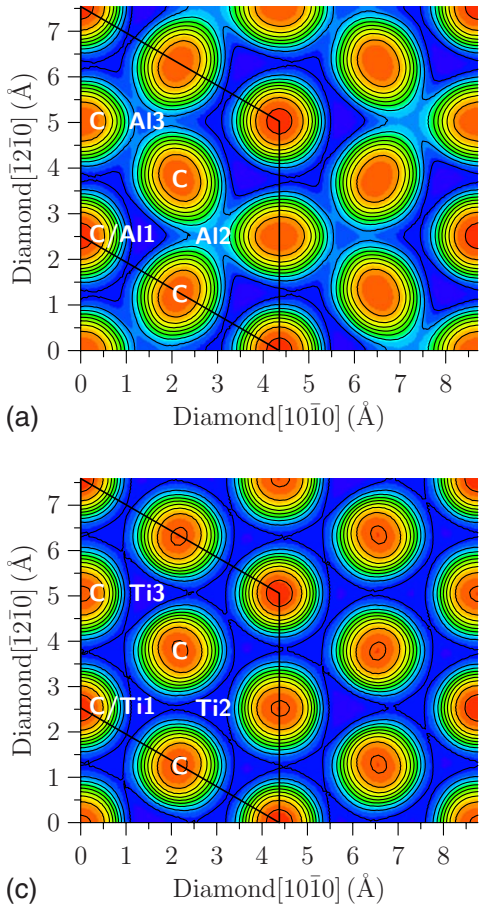

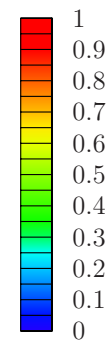

(b)

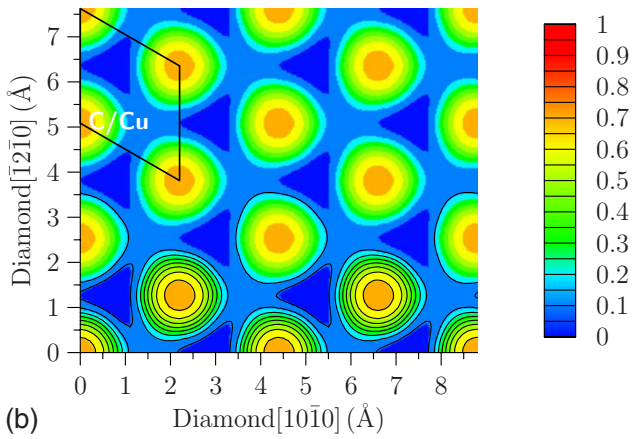

(d)

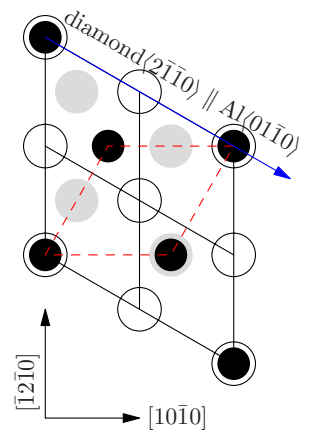

FIG. 2. (Color online) ELF contour plots along the planes that are parallel to the interfaces and between the diamond and the metals. Unit cells are marked by black lines. The positions of atoms at the interfaces are projected on the planes and marked by the element names in the unit cells. The numbers in diamond/Al and diamond/Ti represent the three $\mathrm{Al}$ and $\mathrm{Ti}$ atoms that match four carbon atoms in each unit cell. Each neighboring color represents a difference of 0.1 in ELF. The interface registries of diamond/Al and diamond/Ti interfaces are shown in (d) to assist the visualization. In (d), hollow circles are the first layer of $\mathrm{C}$ atoms; the filled gray circles are the second layer of $\mathrm{C}$; the filled black circles are the first layer of metal atoms. The dashed line marks the plane unit cell of the metal. The arrow shows that the $\langle 2 \overline{1} \overline{1} 0\rangle$ direction of diamond(111) plane is parallel to the $\langle 01 \overline{1} 0\rangle$ direction of $\mathrm{Al}(111)$ or $\mathrm{Ti}(0002)$ plane.

From the above analysis to the ELF contour plots, we found that the order of the bond strength at the diamond/ metal interface is $\mathrm{Ti}-\mathrm{C}>\mathrm{Al}-\mathrm{C}>\mathrm{Cu}-\mathrm{C}$, which is consistent with the order of the work of separation at these interfaces. To form a covalent bond at the diamond/metal interfaces, valence electrons must be shared among bonding atoms. Thus, the bond strength can be estimated from the number of the participating valence electrons that form the covalent carbon-metal bonds. Each carbon atom at a diamond(111) surface has only one dangling bond. On the other side, $\mathrm{Cu}$ has one valence electron, $\mathrm{Al}$ atom has three, and $\mathrm{Ti}$ has four. However, for metals, not all the valence electrons are available to form covalent bonds with carbon atoms at the interface, because free electrons are required to maintain the metallic bonds. Therefore, each $\mathrm{Cu}$ atom provides less than one valence electron, and forms a relatively weaker covalent bond with the carbon atom directly below it. $\mathrm{Al}$ and $\mathrm{Ti}$ can provide one valence electron to develop a stronger covalent bond with carbon atoms directly below them in type (1) bond. In type (2) bonds, each metal atom is shared by 1.5 carbon atoms in average. Thus both $\mathrm{Al}$ and $\mathrm{Ti}$ can provide enough electrons to form both type (1) and type (2) bonds, while maintaining the metallic bonds with the bulk phase. Since Ti can provide more valence electrons than Al, it develops overall stronger interfacial covalent bonds with carbon atoms than Al. This simple estimation is consistent with the order of interface strengths of the diamond/metal interfaces.

\section{Electronic structure of the weakened metal phase}

The charge density distribution and, even more informatively, the change in the charge density reveal how the interface bonding is formed. The charge density difference is calculated as $\Delta \rho(\vec{r})=\rho_{\text {int }}(\vec{r})-\rho_{M}(\vec{r})-\rho_{C}(\vec{r})$, where the subscripts int, $M$, and $C$ stand for the equilibrium interface system, the metal slab, and the diamond slab, respectively. All the atoms are frozen at their equilibrium positions as in the interface models for all the charge density calculations.

As in Fig. 3, the $\Delta \rho(\vec{r})$ for the three interfaces are shown as contour plots, where the red (dark gray) regions are for electron gain, and the blue (light gray) for electron loss. Figure 3 shows that the charge density differences, both gain and loss, reach maxima at the interface region. The maximum electron gain is between the metal atom and the carbon atom right below it, corresponding to type (1) bond. In the interfaces of diamond/Al and diamond/Ti, the carbon atoms that have no metal atom on top of them attract electrons from nearby regions to form shared electrons with the metal atoms on the hollow sites, corresponding to type (2) bonds. The charge gain and loss upon the interface formation go beyond the first metal layer into the metal phase. The second layer of $\mathrm{Al}$ and $\mathrm{Cu}$ and even the third layer of $\mathrm{Ti}$ atoms also show some electronic structure change. The charge density differences approach almost zero at the regions about two to three metal layers away from the interfaces.

In addition to the contour plots in Fig. 3, which show the charge density differences on the cross sections that are perpendicular to the interfaces, we also summarize in Fig. 4 the 

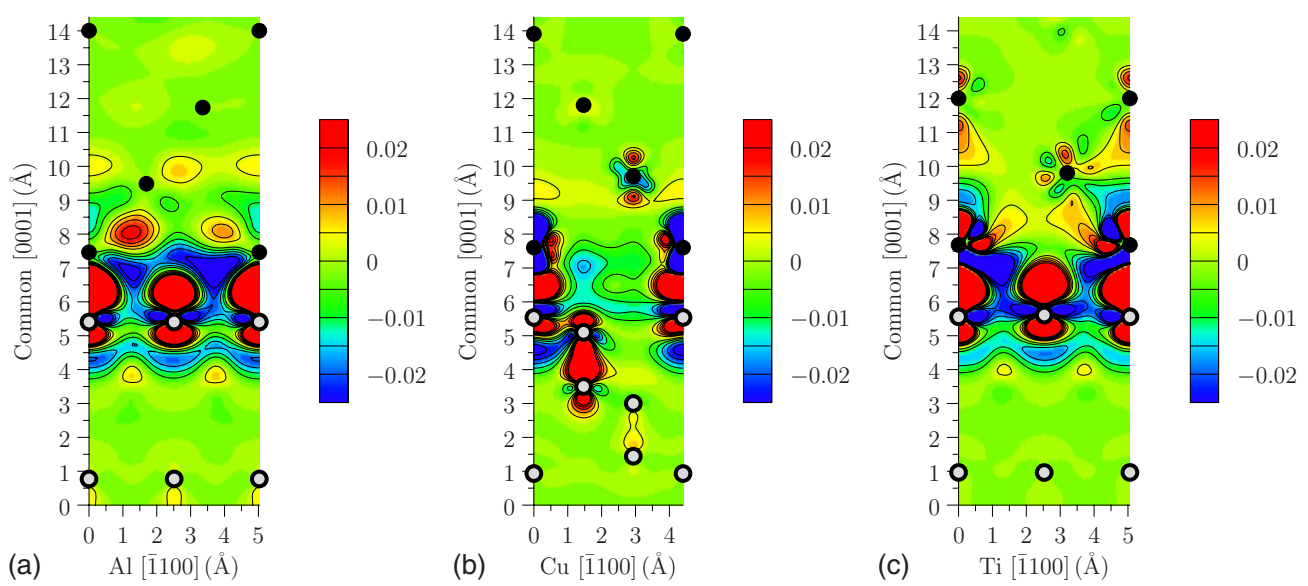

FIG. 3. (Color online) Contour plots of charge density differences of (a) diamond/Al, (b) diamond/Cu, and (c) diamond/Ti interfaces along the metal (1 $\overline{1} 00$ ) planes. The filled gray circles are carbon atoms, and the filled black circles in (a), (b), and (c) are $\mathrm{Al}, \mathrm{Cu}$, and $\mathrm{Ti}$ atoms, respectively. Atoms that are not on the sample planes are not shown. The numbers in the bar are in unit of e/ $\AA^{3}$.

statistical information (the average and the variance) of the charge density differences on each plane that is parallel to the interface. The average value is the net charge transfer in or out of the given plane, and the variance represents the inhomogeneity of charge density difference within the plane. From Fig. 4 we see that the averages and variances decrease to about zero at the planes that are two atomic layers away from the interfaces, while fluctuating the most around the interfaces. For the charge density differences at the regions between the first and the second metal layers, the diamond/Al interface has a wavy average and almost zero variance, suggesting an interplanar charge transfer; the diamond/Ti interface shows almost zero average but a peak of variance, suggesting mainly intraplanar charge transfer; and the diamond/Cu interface has both nonzero average and variance, which appears to have the most inhomogeneous charge transfer. As the average and variance approach to about zero beyond the second metal layer, the influence of the diamond/metal interface is expected to extend to the second metal layer, indicating that range of the interface region.

\section{The location of fracture interface region}

Under an applied load, a fracture may occur right at the interface or inside the bulk phase; thus it is important to
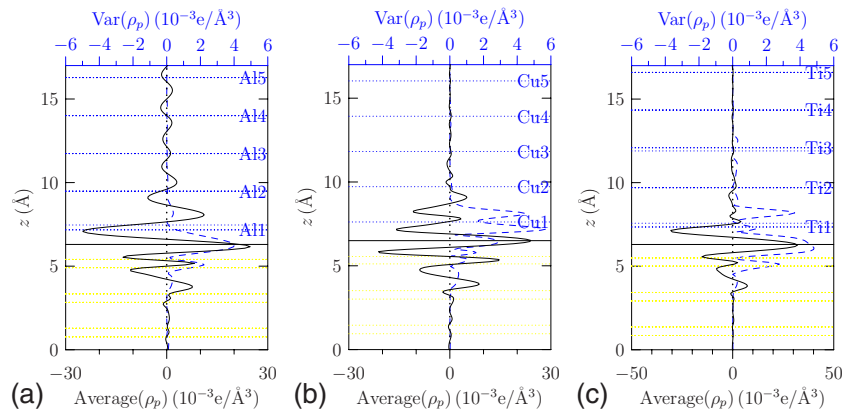

FIG. 4. (Color online) (a) Planewise charge density differences of (a) diamond/Al, (b) diamond/Cu, and (c) diamond/Ti interfaces. The metal layers are marked with horizontal dotted lines and sequential numbers counted from the interface. The interfaces are marked by horizontal solid lines. The black continuous curves are the averages, and the dashed curves are the variances. compute the work of decohesion, $W_{\text {dec }}$, inside the bulk phase and compare it with $W_{\text {sep }}$ to find out the most vulnerable sites for the fracture in the system. $W_{\mathrm{dec}}$ is defined as the energy difference (per unit surface area) between the fractured system and the interface structure, especially when the fracture occurs inside one phase. When the fracture occurs right at the interface, the same definition is referred to as $W_{\text {sep. }}$ The overall strength of the interface region is determined by the weakest link. Because diamond is stronger than metals, only the $W_{\text {dec }}$ inside the metal is compared to $W_{\text {sep }}$ at the interface. If the rupture occurs inside the metal, the $W_{\text {dec }}$ should be simply two times of the surface energy, $2 \gamma_{s}$. However, we have observed apparent deviations when the rupture occurs near the diamond/metal interface. The deviations originated from the chemical interaction at the interface, as observed from the charge transfer analysis in Sec. III C. The charge transfer indicated that the interface is a region that includes at least two atomic layers.

We have calculated the $W_{\text {dec }}$ for the fracture between the first and the second atomic metal layers counted from the interface (represented by $1 \mid 2$ ), between the second and the third $(2 \mid 3)$, and between the third and the fourth (3|4) metal layers. These values are also listed in Table II, in comparison to $W_{\text {sep }}$ and $2 \gamma_{s}$. For all the three metals, the $W_{\text {dec }}$ is much smaller than $W_{\text {sep }}$, meaning the fracture is more likely to occur inside the metal. It is noted that $W_{\mathrm{dec}}(2 \mid 3)$ is the smallest compared to the decohesion energies between other metal layers. There is a difference between $\mathrm{Cu}$ and the other two metals. For $\mathrm{Cu}$, the $W_{\mathrm{dec}}$ at (1|2) and (2|3) are the same. On the other hand, the $W_{\text {dec }}$ at $(1 \mid 2)$ is larger than (2|3) by 0.69 and $0.30 \mathrm{~J} / \mathrm{m}^{2}$ for $\mathrm{Al}$ and $\mathrm{Ti}$, respectively. This means that a fracture should initiate between the second and third atomic layers from the diamond/metal interfaces in $\mathrm{Al}$ and $\mathrm{Ti}$, but it is possible to start even at the first layer in $\mathrm{Cu}$. Eventually, the $W_{\text {dec }}$ converges to $2 \gamma_{s}$ when the influence of the interface diminishes. The weakening effect on the metal phase near the interface can be attributed to the depletion of electrons from the metal phase to the interface. This weakening effect vanishes at regions that are three atomic layers away from the interface due to the vanishing electronic in- 


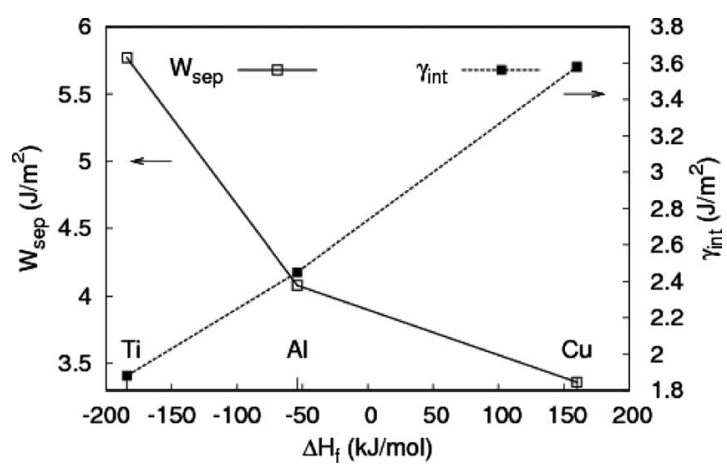

FIG. 5. The ideal works of separation $\left(W_{\text {sep }}\right)$ and interface energies $\left(\gamma_{\text {int }}\right)$ vs formation enthalpies of carbides $\left(\Delta H_{f}\right)$ for the diamond/metal interfaces.

fluence from the diamond/metal interface. For the overall interface region, the smallest $W_{\mathrm{dec}}$ is smaller, but still close to $2 \gamma_{s}$ of the metal phase. Thus to increase the overall strength of the interface region, a stronger metal with a larger surface energy should be considered.

\section{DEPENDENCE OF $W_{\text {sep }}$ AND $\gamma_{\text {int }}$ ON $\Delta H_{f}$}

The carbide formation ability is indicated by the formation enthalpy of the carbide phase, $\Delta H_{f}$. The $\Delta H_{f}$ of the two carbide-forming metals are obtained from literature. It is $-52.4 \mathrm{~kJ} / \mathrm{mol}$ for $\mathrm{Al}_{4} \mathrm{C}_{3}$ per $\mathrm{Al}$ atom, ${ }^{49}$ and $-185.2 \mathrm{~kJ} / \mathrm{mol}$ for TiC per Ti atom. ${ }^{49}$ The $\Delta H_{f}$ of the non-carbide-forming metal, $\mathrm{Cu}$, is calculated to be $160.0 \mathrm{~kJ} / \mathrm{mol}$ per $\mathrm{Cu}$ atom using DFT with an imaginary $\mathrm{CuC}$ of the $\mathrm{NaCl}$ structure. Among all the elements, Ti has the third smallest $\Delta H_{f}$, which is only larger than that of $\mathrm{Hf}\left(\Delta H_{f}=-252.4 \mathrm{~kJ} / \mathrm{mol}\right)^{49}$ and $\mathrm{Zr}\left(\Delta H_{f}=-203.7 \mathrm{~kJ} / \mathrm{mol}\right){ }^{47}$ Plotting $W_{\text {sep }}$ versus $\Delta H_{f}$ in Fig. 5 shows that $W_{\text {sep }}$ decreases with $\Delta H_{f}$. Since the carbide formation ability also decreases with $\Delta H_{f}$, the results suggest that large negative $\Delta H_{f}$ or strong carbide formation ability will improve the strength of the diamond/metal interface.

While $W_{\text {sep }}$ is related to the strength where free surfaces form in fracture, $\gamma_{\text {int }}$ is related to a nucleation process where individual atoms agglomerate on a free surface to form a new interface. $\gamma_{\text {int }}$ is defined as the energy change for the formation of an interface from individual atoms

$$
\gamma_{\text {int }}=\left(E_{12}^{\mathrm{tot}}-N_{M} \mu_{M}-N_{C} \mu_{C}\right) / A,
$$

where $\mu_{M}$ and $\mu_{C}$ are the chemical potentials of the metal atoms in the bulk phases and the carbon atoms in diamond, respectively, $N_{M}$ and $N_{C}$ are the numbers of corresponding atoms in the system, and $A$ is the interface area. The interface energies are listed in Table II and also plotted versus $\Delta H_{f}$ in Fig. 5. The calculated interface energy of diamond $/ \mathrm{Cu}$, $3.58 \mathrm{~J} / \mathrm{m}^{2}$, agrees well with $3.55 \mathrm{~J} / \mathrm{m}^{2}$ in Ref. 16. According to Eq. (1), the $\gamma_{\text {int }}$ and $W_{\text {sep }}$ are correlated with each other by the surface energies. $\gamma_{\text {int }}$ increases with $\Delta H_{f}$ while $W_{\text {sep }}$ decrease with $\Delta H_{f}$. This conclusion is in general agreement with the empirical model, which links increasing interface energies with increasing formation enthalpies, although that model was proposed mainly for transition metals. ${ }^{50}$ The analysis to the relationship between nucleation and $\gamma_{\text {int }}$ will be presented in Sec. V.

\section{ENERGETICAL ANALYSIS OF NUCLEATION}

By nucleation, the change in Gibbs free energy is

$$
\Delta G=V \Delta G_{v}+A^{\prime} \gamma_{N}-A\left(\gamma_{S}-\gamma_{\text {int }}\right),
$$

where $V$ is the volume of the nuclei, $\Delta G_{v}$ is the change in volumetric free energy, $A^{\prime}$ is the area of the nucleus surface, $A$ is the area of the interface, and $\gamma_{S}$ and $\gamma_{N}$ are the surface energies of the substrate and the nuclei, respectively. From Eq. (4) we see that the interface energy $\gamma_{\text {int }}$ is a nucleation barrier. Under a given driving force, the larger the $\gamma_{\text {int }}$ is, the more difficult the nucleation will be. As shown in Fig. 5, $\gamma_{\text {int }}$ increases with $\Delta H_{f}$, which implies that a substrate with a larger negative $\Delta H_{f}$ favors the diamond nucleation. This is reasonable because when $\Delta H_{f}$ is negative, the formation of metal-carbon bonds will release energy and leads to relatively small interface formation energy. The increase in $\gamma_{\text {int }}$ with increasing $\Delta H_{f}$ also implies that the diamond nucleation is difficult and slow on those substrates with a large positive $\Delta H_{f}$.

The interface energy $\left(\gamma_{\text {int }}\right)$ and the surface energies $\left(\gamma_{S}\right.$ and $\gamma_{N}$ ) are the key parameters in determining the growth mode, which also affects the interface adhesion. The layerby-layer Frank-van der Merwe growth mode would form a totally wetted interface and provide the strongest adhesion, and it requires $\gamma_{S}-\gamma_{\text {int }}-\gamma_{N}>0$ or $\gamma_{\text {metal }}-\gamma_{\text {diamond }}>\gamma_{\text {int }}$ in our case. $^{51}$ Apparently, higher metal surface energy and smaller interface energy favor a better wetting interface to enhance the adhesion, and $\mathrm{Ti}$ is the best out of the three metals investigated here. During CVD diamond growth, the growing surface is typically passivated by hydrogen, and the $\gamma_{N}$ for $\mathrm{H}$ terminated diamond (111) is about $\sim 0.03 \mathrm{~J} / \mathrm{m}^{2}$, when the chemical potential of hydrogen is in equilibrium with the source gas of $\mathrm{CH}_{4} \cdot{ }^{52}$ Based on the surface energies listed in Table I and interface energies listed in Table II, we have that the relationship between $\gamma_{\text {metal }}$ $-\gamma_{\text {diamond }}$ and $\gamma_{\text {int }}$ is $0.75-0.03<2.45\left(\mathrm{~J} / \mathrm{m}^{2}\right)$ for $\mathrm{Al}$, $1.28-0.03<3.58\left(\mathrm{~J} / \mathrm{m}^{2}\right)$ for $\mathrm{Cu}$, and $1.92-0.03$ $>1.88\left(\mathrm{~J} / \mathrm{m}^{2}\right)$ for Ti. Thus, among the three metals, only $\mathrm{Ti}$ is possible to form a wetted interface with diamond, evidenced by various experimental data that adherent and tough diamond coatings have been deposited on $\mathrm{Ti}^{27}$ In contrast, the negative values of $\gamma_{\text {metal }}-\gamma_{\text {int }}-\gamma_{\text {diamond }}$ on $\mathrm{Al}$ and $\mathrm{Cu}$ make it difficult to grow adherent diamond coating on the two substrates. To the knowledge of the authors, there is no report on CVD diamond growth on Al. Although the lattice mismatch between diamond and $\mathrm{Cu}$ is only $1.3 \%$, there is no epitaxial growth of diamond on $\mathrm{Cu}$ reported in literature.

Various experiments attempting to deposit diamond on $\mathrm{Cu}$ failed; ${ }^{22-25}$ however, various metal phases, including $\mathrm{Cu}$, can easily grow on diamond. If diamond is the substrate, the requirement of $\gamma_{\text {diamond }}-\gamma_{\text {metal }}>\gamma_{\text {int }}$ for the layer-by-layer growth mode is readily fulfilled because the surface energy of bare diamond surfaces without passivation is as high as $5 \mathrm{~J} / \mathrm{m}^{2}$. This requirement is satisfied for all the three metals tested here and probably for many other metals. So $\mathrm{Cu}$ can be deposited on the diamond substrate, but not the other way around. $^{6,29}$ 


\section{SUMMARY}

In summary, our study suggests that the smaller formation enthalpy of carbide, $\Delta H_{f}(\mathrm{Ti}<\mathrm{Al}<\mathrm{Cu})$, and the larger surface energy, $\gamma_{s}(\mathrm{Ti}>\mathrm{Cu}>\mathrm{Al})$, are required for a metallic interlayer to enhance the adhesion of diamond coatings. The diamond/metal interfaces are strong due to the covalent bonds and the ideal interface strength or the $W_{\text {sep }}$ increases with carbide formation ability, indicated by $\Delta H_{f}$. Upon the formation of the diamond/metal interface, the change in the electronic structure extends to the second atomic layer inside the metal phase. The electron depletion weakens the metal phase, and the fracture location of the overall interface region is inside the metal phase at the second atomic layer from the interface. Thus to increase the overall strength of the interface region, a stronger metal with a larger $\gamma_{s}$ should be considered. During diamond nucleation, the interface energy $\gamma_{\text {int }}$, which is a nucleation barrier for the diamond coating, decreases with $\Delta H_{f}$. Thus smaller $\Delta H_{f}$ promotes diamond nucleation. Metals with large $\gamma_{s}$ and small $\gamma_{\text {int }}$ favor wetting of diamond nuclei, which also contribute to the formation of a well adhered interface. Among the three metals, only Ti can be wetted by diamond nuclei. For all the reasons above, $\mathrm{Ti}$ is the best interlayer material among the three metals considered in the present study. Overall, the study is helpful for materials selection of substrates for CVD diamond coatings. Although the model uses rather ideal sharp interfaces, without considering any carbide phase even on carbide-forming substrates, the conclusions are still valid to serve as theoretical limits for the metallic interlayer selection for diamond coatings.

\section{ACKNOWLEDGMENTS}

The authors would like to appreciate Dr. Xingcheng Xiao's insightful discussions, and the support from Dr. Anil Sachdev and Dr. Paul Krajewski. The calculations are performed at the GM High Performance Computer Center and the USC High Performance Computing Group.

${ }^{1}$ R. S. Balmer, I. Friel, S. M. Woollard, C. J. H. Wort, G. A. Scarsbrook, S. E. Coe, H. El-Hajj, A. Kaiser, A. Denisenko, E. Kohn, and J. Isberg, Philos. Trans. R. Soc. London, Ser. A 366, 251 (2008).

${ }^{2}$ A. De Sio, J. Achard, A. Tallarie, R. S. Sussmann, A. T. Collins, F. Silva, and E. Pace, Appl. Phys. Lett. 86, 213504 (2005).

${ }^{3}$ Y. Fu, H. Du, and J. Miao, J. Mater. Process. Technol. 132, 73 (2003).

${ }^{4}$ S. Srinivasan, J. Hiller, B. Kabius, and O. Auciello, Appl. Phys. Lett. 90, 134101 (2007)

${ }^{5}$ O. Auciello, J. Birrel, J. A. Carlisle, J. E. Gerbi, X. Xiao, B. Peng, and H. D. Espinosa, J. Phys.: Condens. Matter 16, R539 (2004).

${ }^{6}$ P. K. Baumann, T. P. Humphreys, R. J. Nemanich, K. Ishibashi, N. R. Parikh, L. M. Porter, and R. F. Davis, Diamond Relat. Mater. 3, 883 (1994).

${ }^{7}$ X. Xiao, B. W. Sheldon, Y. Qi, and A. K. Kothari, Appl. Phys. Lett. 92,
131908 (2008).

${ }^{8}$ Y. Qi, B. W. Sheldon, H. B. Guo, and A. K. Kothari, Phys. Rev. Lett. 102, 056101 (2009).

${ }^{9}$ H. Liu and D. S. Dandy, Diamond Chemical Vapor Deposition: Nucleation and Early Growth Stages (Noyes, Park Ridge, NJ, 1995), pp. 92-95.

${ }^{10}$ M. Nesladek, K. Vandierendonck, C. Quaeyhaegens, M. Kerkhofs, and L. M. Stals, Thin Solid Films 270, 184 (1995).

${ }^{11}$ D. E. Packham, Handbook of Adhesion (Bath, Avon, 1992), pp. 18-24 and 408-409.

${ }^{12}$ A. Bagchi and A. G. Evans, Thin Solid Films 286, 203 (1996).

${ }^{13}$ F. R. Brotzen, Int. Mater. Rev. 39, 24 (1994).

${ }^{14}$ M. J. Allen and S. D. Senturia, J. Adhes. 25, 303 (1988).

${ }^{15}$ Y. Qi, L. G. Hector, Jr., N. Ooi, and J. B. Adams, Surf. Sci. 581, 155 (2005).

${ }^{16}$ X.-G. Wang and J. R. Smith, Phys. Rev. Lett. 87, 186103 (2001).

${ }^{17}$ M. Lane, Annu. Rev. Mater. Res. 33, 29 (2003).

${ }^{18}$ I. G. Batyrev, A. Alavi, and M. W. Finnis, Phys. Rev. B 62, 4698 (2000).

${ }^{19}$ A. G. Evans, J. W. Hutchinson, and Y. Wei, Acta Mater. 47, 4093 (1999).

${ }^{20}$ Q. H. Fan, E. Pereira, and J. Gracio, J. Mater. Sci. 34, 1353 (1999).

${ }^{21}$ J. Nayaran, V. P. Godbole, G. Matera, and R. K. Singh, J. Appl. Phys. 71, 966 (1992)

${ }^{22}$ L. Constant and F. Le Normand, Diamond Relat. Mater. 6, 664 (1997).

${ }^{23}$ L. Constant, C. Speisser, and F. Le Normand, Surf. Sci. 387, 28 (1997).

${ }^{24}$ N. Jiang, C. L. Wang, J. H. Won, M. H. Joen, Y. Mori, A. Hatta, T. Ito, T. Sasaki, and A. Kiraki, Appl. Surf. Sci. 117/118, 587 (1997).

${ }^{25}$ T. P. Ong, F. Xiong, R. P. H. Chang, and C. W. White, J. Mater. Res. 7, 2429 (1992).

${ }^{26}$ M. Vedawyas, G. Sivananthan, and A. Kumar, Mater. Sci. Eng., B 78, 16 (2000).

${ }^{27}$ M. D. Drory and J. W. Hutchinson, Science 263, 1753 (1994).

${ }^{28}$ H. Kawarada, Surf. Sci. Rep. 26, 205 (1996).

${ }^{29}$ P. K. Baumann and R. J. Nemanich, Phys. Rev. B 58, 1643 (1998).

${ }^{30}$ M. Kasu, K. Ueda, H. Kageshima, and Y. Yamauchi, Diamond Relat. Mater. 17, 741 (2008).

${ }^{31}$ Y. Qi and L. G. Hector, Jr., Phys. Rev. B 69, 235401 (2004)

${ }^{32}$ G. Kresse and J. Hafner, Phys. Rev. B 49, 14251 (1994).

${ }^{33}$ G. Kresse and J. Furthmüller, Comput. Mater. Sci. 6, 15 (1996).

${ }^{34}$ G. Kresse and J. Joubert, Phys. Rev. B 59, 1758 (1999).

${ }^{35}$ J. P. Perdew, J. A. Chevary, S. H. Vosko, K. A. Jackson, M. R. Pederson, and D. J. Singh, Phys. Rev. B 46, 6671 (1992).

${ }^{36}$ J. L. F. Da Silva, F. Stampfl, and M. Scheffler, Surf. Sci. 600, 703 (2006).

${ }^{37}$ H. M. Polatoglou, M. Methfessel, and M. Scheffler, Phys. Rev. B 48, 1877 (1993).

${ }^{38}$ Z. Xiong, S. Shi, C. Ouyang, M. Lei, L. Hu, Y. Ji, Z. Wang, and L. Chen, Phys. Lett. A 337, 247 (2005).

${ }^{39}$ Ž. Crljen, D. Šokčević, R. Brako, and P. Lazić, Vacuum 71, 101 (2003).

${ }^{40}$ Th. Rodach, K.-P. Bohnen, and K. M. Ho, Surf. Sci. 286, 66 (1993).

${ }^{41}$ H. L. Skriver and N. M. Rosengaard, Phys. Rev. B 46, 7157 (1992).

${ }^{42}$ D. E. Jiang and E. A. Carter, Acta Mater. 53, 4489 (2005).

${ }^{43}$ D. J. Siegel, L. G. Hector, and J. B. Adams, Phys. Rev. B 67, 092105 (2003).

${ }^{44}$ A. Mattoni, L. Colombo, and F. Cleri, Phys. Rev. Lett. 95, 115501 (2005).

${ }^{45}$ J. P. Hirth and J. R. Rice, Metall. Trans. A 11, 1501 (1980).

${ }^{46}$ J. R. Rice, J. Mech. Phys. Solids 26, 61 (1978).

${ }^{47}$ D. Becke and K. E. Edgecombe, J. Chem. Phys. 92, 5397 (1990).

${ }^{48}$ B. Silvi and A. Savin, Nature (London) 371, 683 (1994).

${ }^{49}$ D. R. Lite, CRC Handbook of Chemistry and Physics, 71st ed. (CRC, Boca Raton, FL, 1991), pp. 5-18, 5-35, 5-56, and 5-59.

${ }^{50}$ F. R. de Boer, R. Boom, W. C. M. Mattens, A. R. Miedema, and A. K. Niessen, Cohesion in Metals. Transition Metal Alloys (North-Holland, Amsterdam, 1989).

${ }^{51}$ S. A. Chambers, Surf. Sci. Rep. 39, 105 (2000).

${ }^{52}$ S. Hong and M. Y. Chou, Phys. Rev. B 57, 6262 (1998). 